\title{
Palliative Treatment of Locally Advanced Non Metastatic Lung Cancer
}

\author{
Gael Kietga, Wilfried Mosse, Patricia Agbanglanon, Bertrand Compaore, Davy Nchepo, \\ Evrard Seka, Sanae Elmajjaoui, Hanane Elkacemi, Tayeb Kebdani, Amine Lachgar, \\ Noureddine Benjaafar
}

Radiotherapy Department, National Institute of Oncology, Rabat, Morocco

Email: gaelkietga@yahoo.fr

How to cite this paper: Kietga, G., Mosse, W., Agbanglanon, P., Compaore, B., Nchepo, D., Seka, E., Elmajjaoui, S., Elkacemi, H., Kebdani, T., Lachgar, A. and Benjaafar, N. (2021) Palliative Treatment of Locally Advanced Non Metastatic Lung Cancer. Journal of Cancer Therapy, 12, 71-77. https://doi.org/10.4236/jct.2021.122008

Received: November 24, 2020

Accepted: February 6, 2021

Published: February 9, 2021

Copyright $\odot 2021$ by author(s) and Scientific Research Publishing Inc. This work is licensed under the Creative Commons Attribution International License (CC BY 4.0).

http://creativecommons.org/licenses/by/4.0/

\begin{abstract}
Introduction: To determine the proportion and the reasons which lead to palliative treatment in patients initially a candidate for concomitant chemoradiotherapy (CCRT). Methods: A retrospective study including patients followed for locally advanced lung cancer newly diagnosed from April 1, 2016, to $12 / 31 / 2017$ in the radiotherapy department of the National Oncology Institute who received palliative treatment. Results: We collected 52 patients out of a total of 225 stage III patients (23\%) followed by lung cancer candidates for CCRT who had undergone palliative treatment. The mean age in our series was 61.23 years [22 - 81] with $86 \%$ male. The majority of patients (71\%) had Performance Status (PS) $\leq 2$. Histological confirmation was obtained by pathological examination in all our patients. It was an adenocarcinoma (ADK) in $54 \%$ of cases; squamous cell carcinoma in $46 \%$ of cases. The reasons for palliative treatment were mainly due to dosimetric constraints: large tumor volume 22/52 (42\%); the tumor location close to the bone marrow in 15 of 52 (29\%) patients; and general Performance Status impairment (29\%) in 15 of 52 patients. Palliative treatment consisted of palliative chemotherapy in 37 of 52 patients (71\%), among whom 19 (51\%) were stable after 2 months of chemotherapy, in palliative dose chest radiotherapy on the pulmonary parenchyma and/or mediastinum in 10 of $52(19 \%)$ patients, and supportive care in $5(10 \%)$ patients. We observed 40/52 (77\%) cases of stationary course, $04 / 52(8 \%)$ cases of progress to metastases, and $08 / 52(15 \%)$ deaths before radiotherapy. Conclusion: A large proportion of patients followed for locally advanced non-metastatic lung cancer are not eligible for curative treatment. The reasons for the palliative treatment of patients followed for lung cancer candidates for CCRT are variable but for a large proportion of patients due to the deterioration of their state of health during their diagnostic journey. Hence, there is the need to improve the early diag-
\end{abstract}


nosis and early management of patients with lung cancer to avoid delayed care.

\section{Keywords}

Lung Cancer, Palliative Treatment, Locally Advanced

\section{Introduction}

Bronchopulmonary cancer is the first cancer in the world in terms of incidence (11.6\% of total cases) for both sexes. It is also the leading cause of cancer death worldwide (18.4\% of total cancer deaths) [1]. Bronchopulmonary cancer is a heterogeneous group of tumors. At presentation, the most common clinical manifestations are cough, hemoptysis, and dyspnea. Every patient with suspected lung cancer should undergo computed tomography (CT) scan of the chest and upper abdomen to evaluate the extent of the primary tumor and potential spread. The diagnosis is made based upon histopathologic (e.g., tissue biopsy) specimens or in some cases the pathologic evaluation of cytologic (e.g., pleural fluid). The $8^{\text {th }}$ edition of the Tumor Node Metastasis (TNM) is used for staging of bronchopulmonary cancer. Concomitant chemoradiotherapy (CCRT) is the recommended standard treatment in patients with locally advanced lung cancer [2]. However, a significant proportion of her patients who are candidates for CCRT will eventually receive palliative treatment. This study aims to determine the proportions, reasons and modalities of palliative treatment in patients with locally advanced non-metastatic lung cancer in our institute.

\section{Methods}

We performed a retrospectively review of the consultation register containing the list of patients with locally advanced non-metastatic lung cancer, whose indication for CCRT at a curative dose had been selected; and referred to the radiotherapy department of the national... for irradiation with curative intent from 04/04/2016 to $31 / 12 / 2017$.

We then obtained a list of 225 patients. Among his patients, we selected those who did not ultimately benefit from a curative dose CCRT $(n=52)$ that we included in our study. Data collection was carried out through the use in addition to clinical patient records, the consultation appointment register, and the simulation scanner programming and treatment registers in the radiotherapy department. The diagnosis was confirmed pathologically in all cases by image guidance cytology or biopsy, bronchio-alveolar lavage and classified according to Word Health Organisation WHO histological classification of lung cancer staging was done according to AJCC staging system, 8th edition based on the available clinical and radiological findings. The clinical records of the patients were reviewed in relation with age, sex, pathological report, stage, treatment reasons and treatment modalities. 
PALLIATIVE TREATMENT: Treatment given to relieve the symptoms and reduce the suffering caused by cancer and other life-threatening diseases.

In our study, Palliative treatment included:

- Palliative radiotherapy with a lower doses of radiation with the aims to shrink cancer, slow down its growth or control symptoms. It doesn't aim to cure cancer and can be safely used without much in the way of side effects for the patient.

- Palliative chemotherapy: chemotherapy cannot cure the cancer. However, chemotherapy may be helpful in shrinking the cancer, improving or completely eliminating distressing symptoms caused by the cancer for a period of time and helping you live longer.

- Support Care: In our study refers to patients who do not benefit from specific palliative cancer treatment such as palliative dose radiotherapy or palliative chemotherapy. Its patients benefit exclusively from symptomatic treatment and comfort care to improve their quality of life.

\section{Exclusion Criteria:}

- Patients treated by CCRT curative dose

- Metastasis lung cancer

Descriptive statistics were used for describing the data using SPSS version 22 and results were presented in percentage and simple frequency.

\section{Results}

We collected 52 patients out of a total of 225 stage III patients (23\%) followed by lung cancer candidates for CCRT who had undergone palliative treatment. The mean age in our series was 61.23 years [22 - 81] with $86 \%$ male; the majority of patients $(71 \%)$ had Performance Status (PS) $\leq 2$. Histological confirmation was obtained by pathological examination in all our patients. It was an adenocarcinoma $(\mathrm{ADK})$ in $54 \%$ of cases; squamous cell carcinoma in $46 \%$ of cases (Table 1 ).

The reasons for palliative treatment were mainly due to dosimetric constraints: large tumor volume 22/52 (42\%); the tumor location close to the bone marrow in 15 of 52 (29\%) patients; and general World Health Organisation (WHO) Performance Status (PS) impairment (29\%) in 15 of 52 patients (Table 2).

Palliative treatment consisted of palliative chemotherapy alone in 35 of 52 patients (67\%); among whom $19(54,28 \%)$ were stable after 02 months of chemotherapy, in palliative dose chest radiotherapy alone on the pulmonary parenchyma and/or mediastinum in 08 of $52(15 \%)$ patients, and supportive care in $5(10 \%)$ patients; palliative radiotherapy + palliative chemotherapy in 4 (8\%) (Table 3$)$.

Table 1. Patient characteristics.

\begin{tabular}{cc}
\hline Average (year) & $61.23[22-81]$ \\
\hline Histological Type (\%) & \\
Adenocarcinoma & $\mathrm{n}=28(54)$ \\
Squamous cell carcinoma & $\mathrm{n}=24(46)$ \\
\hline
\end{tabular}


Table 2. Reasons for palliative treatment.

\begin{tabular}{cccc}
\hline Reasons & Large tumor size & $\begin{array}{c}\text { Localization close } \\
\text { to the cord }\end{array}$ & $\begin{array}{c}\text { Alteration of WHO } \\
\text { performance status }\end{array}$ \\
\hline $\mathrm{n}(\%)$ & $22(42)$ & $15(29)$ & $15(29)$ \\
\hline
\end{tabular}

Table 3. Modalities of palliative treatment.

\begin{tabular}{cc}
\hline Processing Modalities & $\mathrm{n}(\%)$ \\
\hline Palliative chemotherapy alone & $35(67)$ \\
Palliative radiotherapy only & $8(15)$ \\
Supportive care & $5(10)$ \\
Palliative radiotherapy + palliative chemotherapy & $4(8)$ \\
\hline
\end{tabular}

We observed $40 / 52(77 \%)$ cases of stationary course, $04 / 52$ (8\%) cases of progress to metastases, and 08/52 (15\%) deaths before radiotherapy.

\section{Discussion}

In our study, 23\% (52/225) of patients candidates for CCRT for the treatment of a locally advanced lung cancer had undergone palliative treatment. AG Robinson and Collaborators in a series of 237 patients treated between 2008 and 2012 for locally advanced stage III non-small cell lung cancer reported that 130 patients (55\%) among the candidates for CCRT had not received treatment [3]. Another series, that of Shamzi and colleagues in another study of 122 patients followed for locally advanced lung cancer found that $50 \%$ of patients had undergone palliative treatment [4]. These higher rates compared to our study could be explained by the larger study population, the longer inclusion time, and the variation in socio-demographic characteristics, in particular the age between the 2 studies populations. Thereby, the median age of the patients in our series was 61.23 years in the literature younger than in Robinson and Shamsi series with a median age of 68 years and 70 years respectively [22 - 81]; collaborator found an average age of 68 years in Ontario [3] and found an average age of 70 years [4].

The reasons for the palliative treatment of lung cancer in our center were essentially due essentially to dosimetric constraints: large tumor volume; localization close to the spinal cord marrow and the deterioration of performance status $(>2)$ and pulmonary function of our patients. Thus, these reasons are comparable to those found by Sundstrom and collaborators in a study involving $421 \mathrm{pa}-$ tients with locally advanced stage III or IV lung tumors treated by palliative radiotherapy, who included patients with disease too advanced for inoperable curative radiotherapy, those with chest symptoms due to a central tumor threatening the airways [5]. The third international consensus on palliative radiotherapy and symptom control had defined the following factors as intervening in the decision to administer palliative radiotherapy rather than curative: the general 
condition of the patient, the type of tumor, the stage, pulmonary function, volume to be irradiated, symptoms, weight loss and patient choice [6]. Its reasons are comparable to the 2013 American College of Chest Physicians (ACCP) recommendations which suggest treatment with palliative radiotherapy for stage III patients; with a performance score of 3 - 4 in the ECOG scale; with major comorbidities and tumors too large to benefit from curative treatment [7].

In our study, palliative treatment of locally advanced non-metastatic lung cancer consisted of palliative chemotherapy; supportive care, or palliative radiotherapy. This result is comparable to that found by Rijke and collaborators in the Netherlands, who in a series of 66 patients treated for stage IIIA lung cancer and 68 stage IIIB patients who received non-curative treatment, had benefited from the same methods found in our study that is, palliative radiotherapy; chemotherapy and supportive care [8]. Among its these modalities, palliative radiotherapy were done in represented the palliative treatment of in $19 \%$ of our patients; In a study on the care pathway care i Rabat in 2015, Lachgar and collaborators found a similar rate of $16.98 \%$ of patients treated with palliative radiotherapy [9]. Although we observe a slight increase in the rate of palliative radiotherapy, it could be improved. In our series, $54.28 \%$ of patients $(n=21)$ who received palliative chemotherapy were stable. This result is comparable to that found by HARISON and collaborators who, in a randomized study of 906 patients followed for lung cancer, found between $55 \%$ - $63 \%$ of patients had an improved or stable state after 02 months of palliative chemotherapy. The authors thus concluded that palliative chemotherapy improves the quality of life of patients [10].

O'Rourke and Edwards [11] report a prospective single-center audit of 29 lung cancer patients awaiting radical (potentially curative) radiation therapy. They measured the size of the tumor on diagnostic scans and compared it to the size of planning scans. As a result, $21 \%$ of potentially curable patients became incurable on the waiting list. This study shows that the result is compromised by the waiting times that allow tumor progression. O'Rourke and Edwards [11] report a prospective single-center audit of 29 lung cancer patients awaiting radical (potentially curative) radiation therapy. They measured the size of the tumor on diagnostic scans and compared it to the size of planning scans. As a result, $21 \%$ of potentially curable patients became incurable on the waiting list. This study shows that the result is compromised by the waiting times that allow tumor progression hence the need to improve diagnostic facilities and minimize the waiting time for these patients especially since in our study, $15 \%$ of patients died between the date of their diagnosis and before the date of their radiation therapy treatment.

The retrospective nature and the small number of patient in our study of did not allow us to be exhaustive in the data collection. However, we were able to achieve our goal of determining the proportions, reasons, and treatment modalities of patients monitored for lung cancer who were unable to perform the palliative treatment. 


\section{Conflicts of Interest}

The authors declare no conflicts of interest regarding the publication of this paper.

\section{References}

[1] Bray, F., Ferlay, J., Soerjomataram, I., Siegel, R.L., Torre, L.A. and Jemal, A. (2018) Global Cancer Statistics 2018: GLOBOCAN Estimates of Incidence and Mortality Worldwide for 36 Cancers in 185 Countries. CA: A Cancer Journal for Clinicians, 68, 394-424. https://doi.org/10.3322/caac.21492

[2] Giraud, P., Lacornerie, T. and Mornex, F. (2016) Radiotherapy for Primary Lung Carcinoma. Cancer Radiotherapie, 20, S147-S156.

https://doi.org/10.1016/j.canrad.2016.07.009

[3] Robinson, A.G., Young, K., Balchinmsc, K., Owen, T. and Ashworth, A. (2015) Reasons for Palliative Treatments in Stage III Non-Small-Cell Lung Cancer: What Contribution Is Made by Time-Dependent Changes in Tumour or Patient Status? Current Oncology, 22, 399-404. https://doi.org/10.3747/co.22.2689

[4] Al-Shamsi, H.O., Al Farsi, A. and Ellis, P.M. (2014) Stage III None Small-Cell Lung Cancer: Establishing a Benchmark for the Proportion Patients Suitable for Radical Treatment. Clinical Lung Cancer, 15, 274-280.

[5] Sundstrøm, S., Bremnes, R., Aasebø, U., Aamdal, S., Hatlevoll, R. (2004) Hypofractionated Palliative Radiotherapy (17 Gy per Two Fractions) in Advanced Non-Small-Cell Lung Carcinoma Is Comparable to Standard Fractionation for Symptom Control and Survival: A National Phase III Trial. Journal of Clinical Oncology, 22, 802-810. https://doi.org/10.1200/JCO.2004.06.123

[6] Rodrigues, G., Macbeth, F., Burmeister, B., Kelly, K.-L., Bezjak, A., Langer, C., Hahn, C. and Movsas, B. (2012) Consensus Statement on Palliative Lung Radiotherapy: Third International Consensus Workshop on Palliative Radiotherapy and Symptom. Control Clinical Lung Cancer, 13, 1-5.

https://doi.org/10.1016/j.cllc.2011.04.004

[7] Ramnath, N., Dilling, T.J., Harris, L.J., Kim, A.W., Michaud, G.C., Balekian, A.A., Diekemper, R., Detterbeck, F.C. and Arenberg, D.A. (2013) Treatment of Stage III Non-small Cell Lung Cancer Diagnosis and Management of Lung Cancer. American College of Chest Physicians Evidence-Based Clinical Practice Guidelines CHEST, 143, e314S-e340S.

[8] de Rijkea, J.M., Schoutena, L.J., ten Veldec, G.P.M., Wanders, S.L., Bollene, E.C.M., Lalisangf, R.I., van Dijckg, J.A.A.M., Kramer, G.W.P. and van den Brandt, P.A. (2004) Influence of Age, Comorbidity and Performance Status on the Choice of Treatment for Patients with Non-Small Cell Lung Cancer; Results of a Population-Based Study. Lung Cancer, 46, 233-245.

https://doi.org/10.1016/j.lungcan.2004.03.011

[9] Lachgar, A., Sahli, N., Toulba, A., Kebdani, T. and Benjaafar, N. (2015) Cancer Pulmonaire: Parcours de soins au service de radiothérapie à l'institut national d'oncologie de Rabat. The Pan African Medical Journal, 21, 253.

https://doi.org/10.11604/pamj.2015.21.253.6627

[10] Harrison, L.D., Zhang-Salomonsmsc, J., Mates, M., Booth, C.M., King, W.D. and Mackillop, W.J. (2015) Comparing Effectiveness with Efficacy: Outcomes of Palliative Chemotherapy for Non-Small-Cell Lung Cancer in Routine Practice. Current Oncology, 
22, 184-191. https://doi.org/10.3747/co.22.2419

[11] O’Rourke, N. and Edwards, R. (2000) Lung Cancer Treatment Waiting Times and Tumour Growth. Clinical Oncology, 12, 141-144.

https://doi.org/10.1053/clon.2000.9139 